\title{
A polyimide/cellulose lithium battery separator paper
}

\author{
Shanshan Wang, Chuanshan Zhao*, Wenjia Han, and Yifei Jiang \\ State Key Laboratory of Biobased Material and Green Papermaking, Qilu University of Technology, Jinan, 250353, P. R. China
}

\begin{abstract}
A PI/cellulose composite separator was prepared by adsorbing bacterial cellulose on a PI separator prepared via a papermaking process. The PI/cellulose separator exhibited good thermal stability and electrolyte wetting properties compared to commercial celgard 2340 separator. The amount of bacterial cellulose added was $20 \%$. Bacterial cellulose fills the porosity of the PI separator. The PI/cellulose composite separator possesses excellent thermal stability even at $200{ }^{\circ} \mathrm{C}$ and high electrolyte wettability with a contact angle of $58^{\circ}$.
\end{abstract}

\section{Introduction}

Lithium-ion battery (LIB) has been received more and more attention due to their high specific energy, high energy density, long lifetime cycle, low self-discharge rate, and high operational voltage. They have been widely used in consumer electronics, such as portable computers, camcorders and cell phones. In recent years, their applications have been further expanding into electric vehicles(EV) and hybrid electric vehicles(HEV $)^{[1]}$. The safety issue of battery, however, becomes a critical feature in $\mathrm{EV}$ and $\mathrm{HEV}^{[2]}$. LIBs are mainly composed of a cathode, an anode, an electrolyte, and a separator ${ }^{[3]}$. Separator plays the vital role of safety by preventing physical contact of the cathode and anode electrodes as well as influencing the transfer of lithium ions inside the cell. Current commercial separators for LIBs are porous polyolefin separators, which are made from polyethylene (PE) or/and polypropylene (PP) ${ }^{[4]}$.

In recent years, researchers have made many efforts to develop new materials for battery separators with high thermal stability and high electrolyte wettability. Polyimide (PI) is a new type of special insulating material with high strength, high modulus, high temperature resistance, radiation resistance, and corrosion resistance. It is widely used in various fields. In order to improve the safety of the LIB, the performance requirements of the lithium ion battery for the separator can be better satisfied by coating the surface of the separator with inorganic particles. Xingxing Liang et al. ${ }^{[5]}$ prepared a $\mathrm{SiO}_{2} / \mathrm{Al}_{2} \mathrm{O}_{3}$ PI separator by simply coating a mixture of $\mathrm{Al}_{2} \mathrm{O}_{3}$ and $\mathrm{SiO}_{2}$ on the PI separator. The electrochemical properties of the separator were tested in a lithium ion battery, and the $\mathrm{SiO}_{2} / \mathrm{Al}_{2} \mathrm{O}_{3}$ coated electrospun PI separator exhibited better cycle performance and rate performance. Due to the inherent thermal stability of ceramic materials and PI, the composite separator has high thermal stability. However, the surface coating of an inorganic coating undoubtedly increases the thickness of the separator, which is disadvantageous for battery assembly, and the binder that binds inorganic ions at a high temperature may melt to cause the inorganic particles to fall off. Ying Wang et al. ${ }^{[4]}$ added $\mathrm{SiO}_{2}$ in the process of electrospinning to prepare $\mathrm{SiO}_{2}$-PI separator. The $\mathrm{SiO}_{2}$ particles were completely embedded in the PI separator structure without any binder, and the prepared flexible $\mathrm{SiO}_{2}$-PI separator had excellent electrolyte wettability and enhanced thermal stability. However, Chuan Shi et al. ${ }^{[6]}$ proposed that the PI separator itself has no closed cell properties, and the inorganic particles do not have closed cell properties. In order to improve the safety of the LIB at high temperatures, a PE separator with thermal shutdown properties is coated on the surface of the PI separator. Compared with the PI separator, the PE-PI separator has closed cell performance, and the temperature range of the thermal closed pore performance increased from 120 to $200{ }^{\circ} \mathrm{C}$.

The PI fiber is obtained by spinning a polyamic acid solution. The surface of the PI fiber is smooth and flat, and no splitting phenomenon occurs. Cross-linking and chemical bonding do not occur between the fibers, so that it is necessary to add a binder. Recent composite separators have attracted much attention, however, as they offer excellent thermal stability and wettability by the nonaqueous electrolyte. In this study, a traditional wet papermaking process was used, and plant fibers were added to PI fibers to prepare PI/cellulose composite battery separator paper.

\section{EXPERIMENTAL}

\section{1 materials and instruments}

PI fiber, length $6 \mathrm{~mm}$, fineness $1.67 \mathrm{dtex}$; Softwood fiber; Bacterial cellulose; Enhancer, Polyacrylamide; Silicone oil defoamer; Dispersant, Polyethylene oxide; Adhesive, Polyvinyl alcohol; Tetramethyl piperidine oxide.

*Corresponding author:ppzcs78@163.com 


\subsection{Preparation of $\mathrm{PI} /$ cellulose composite battery separator}

The $90{ }^{\circ} \mathrm{SR}$ softwood fiber and the beaten PI fiber are used to prepare a $50 \mathrm{~g} / \mathrm{m}^{2}$ PI battery separator substrate, wherein the mass ratio of softwood fiber to PI fiber is 1:9. Dispersing agents, defoamers, reinforcing agents, and binders were added during the papermaking process. After uniformly mixing in the fiber disintegrator, a wet papermaking process is used to obtain a PI fiber battery separator. Finally, TEMPO-oxidized bacterial cellulose was coated on the PI battery separator to obtain a PI/ cellulose composite battery separator.

\subsection{PI separator characteristics}

Physical properties of battery separator including thickness, porosity, average pore size, gas permeability, tortuosity, wettability, liquid absorption, heat shrinkage were measured. Electrochemical performance including electrochemical impedance spectroscopy, cycle performance, ionic conductivity, linear volt-ampere scanning test and rate performance were characterized in a multi-channel workstation.

\subsubsection{Pore size distribution}

Lithium-ion battery separators should have a pore size of appropriate diameter, uniform pore distribution and pore size distribution, a range of porosity; uniform pore distribution ensures uniform current density. Generally, the pore size is small enough, in the range of $0.01 \mu \mathrm{m}$ to $0.05 \mu \mathrm{m}$, which can effectively prevent the passage of "lithium dendrites". And the puncture resistance of the separator is good. The safety of the LIB is improved. The pore size of the separator is measured by a separator aperture analyzer.

\subsubsection{Thermal stability}

When the battery is overcharged, short-circuited, or impacted, a large amount of heat will be emitted, and the temperature will rise rapidly. The separator should have the characteristics of maintaining the original size and mechanical strength at a high temperature to prevent the battery from being short-circuited by direct contact between the positive and negative electrodes. The thermal stability of the separator is responsive to the shrinkage of the separator at different temperatures.

\subsubsection{Electrolyte wettability}

The performance of the LIB infiltrating the electrolyte characterizes the charge and discharge capacity of the battery, and the retention of the electrolyte by the separator can increase the lifetime of the LIB.

\section{RESULTS AND DISCUSSION}

\subsection{Physical properties and paper structure}
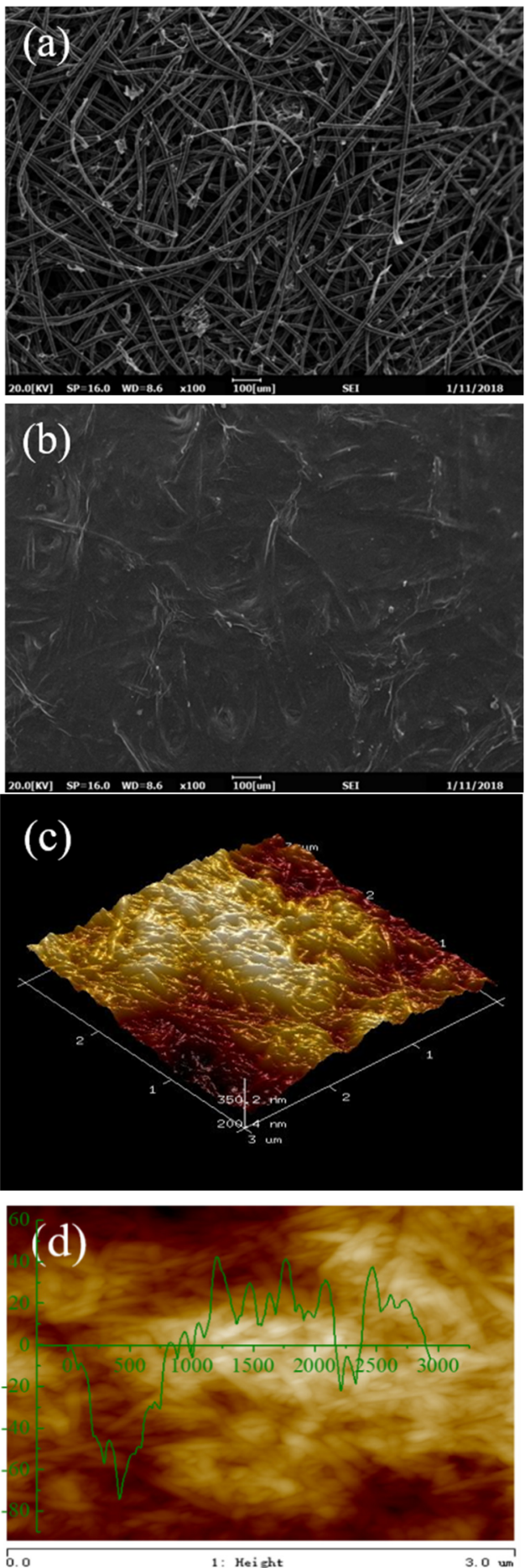

Figure 1. SEM images of PI (a) and PI/cellulose (b) separator. (c, d) AFM images of PI/cellulose separator.

The prepared separator paper was placed in a sealed bag and allowed to stand for $12 \mathrm{~h}$, and the physical properties were measured, as shown in Table 1.

Table 1. Physical properties of different separator types

\begin{tabular}{|c|c|c|c|}
\hline Sample & $\begin{array}{c}\text { Tensile } \\
\text { index } \\
/ \mathbf{N} \bullet \mathbf{m}^{-1} \mathbf{- 1}\end{array}$ & $\begin{array}{c}\text { Electrolyte } \\
\text { uptake } / \%\end{array}$ & $\begin{array}{c}\text { Pore size } \\
/ \boldsymbol{\mu m}\end{array}$ \\
\hline $\begin{array}{c}\text { Celgard } \\
2340\end{array}$ & 242.09 & 60.23 & 0.05 \\
\hline
\end{tabular}




\begin{tabular}{|c|c|c|c|}
\hline PI & 17 & 249.04 & 52.63 \\
\hline PI/cellulose & 27.07 & 108.72 & 21.21 \\
\hline
\end{tabular}

Table 1 shows the physical properties of the different types of separator paper. As can be seen from Table 1, the pore diameter of the $\mathrm{PI} /$ cellulose composite separator paper is reduced, the tensile index of the PI/ cellulose separator, and the liquid absorption rate increased. Bacterial cellulose fibers were coated on the surface of PI network. Cellulose is filled between the pores of the PI fibers to form a dense structure, so that the surface of the paper became smooth, as shown in Figure 1. In addition, the purity of cellulose is as high as $99 \%$, and the hydrogen bonding between cellulose increases the tensile strength of the paper. Since cellulose contains a large amount of hydroxyl groups, it has water absorption, can improve the electrolyte absorption of the separator, and prevent the lithium battery's self-discharge phenomenon.

\subsection{Pore size and Electrolyte wettability}

The pore size and porosity of the PI fiber separator paper were measured using a separator aperture analyzer.

The pore diameter of the PI/cellulose separator prepare from the pulp-treated PI fiber-added softwood fiber and bacterial cellulose decreased from 52.63 to $21.21 \mu \mathrm{m}$. The pore size distribution and distribution of the separator are shown in Figure 2. As shown in Figure 2a, the pore size distribution of the PI separator is between 50 and $60 \mu \mathrm{m}$, with an average value of $52.63 \mu \mathrm{m}$. As shown in Figure $2 \mathrm{~b}$, the $\mathrm{PI} /$ cellulose separator is mainly distributed in the range of 16 to $25 \mu \mathrm{m}$, and the average pore diameter is $21.21 \mu \mathrm{m}$. Since the length of the cellulose fiber is much shorter than that of the PI fiber, cellulose fibers fills the pores in PI network between the PI fibers, forming a compact structure with smaller pores distributed in the separator, which is favorable to the absorption of electrolyte. The lithium ion conductivity of the lithium battery separator effectively prevents self-discharge of the battery. Figure $2 \mathrm{c}$ shows that a electrolyte droplet is rapidly absorbed when contacting the separator, which is related to the larger pore size of the PI separator. As shown in Figure 2d, the PI/cellulose has a contact angle of $58^{\circ}$, possessing good electrolyte wettability.

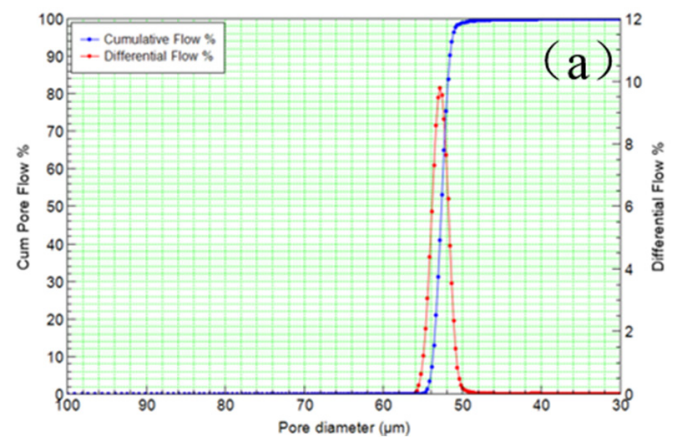

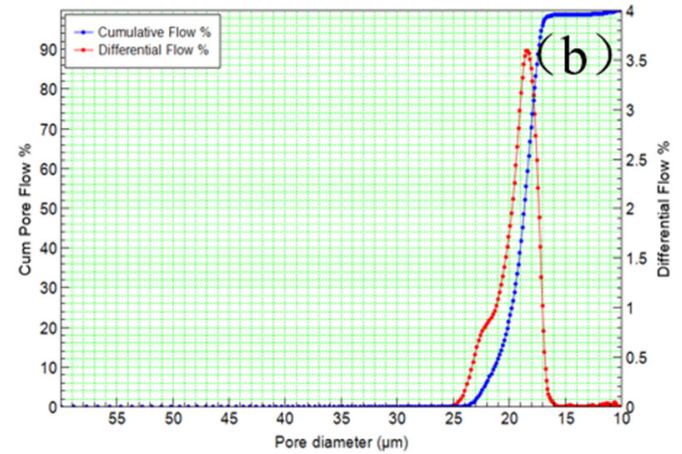

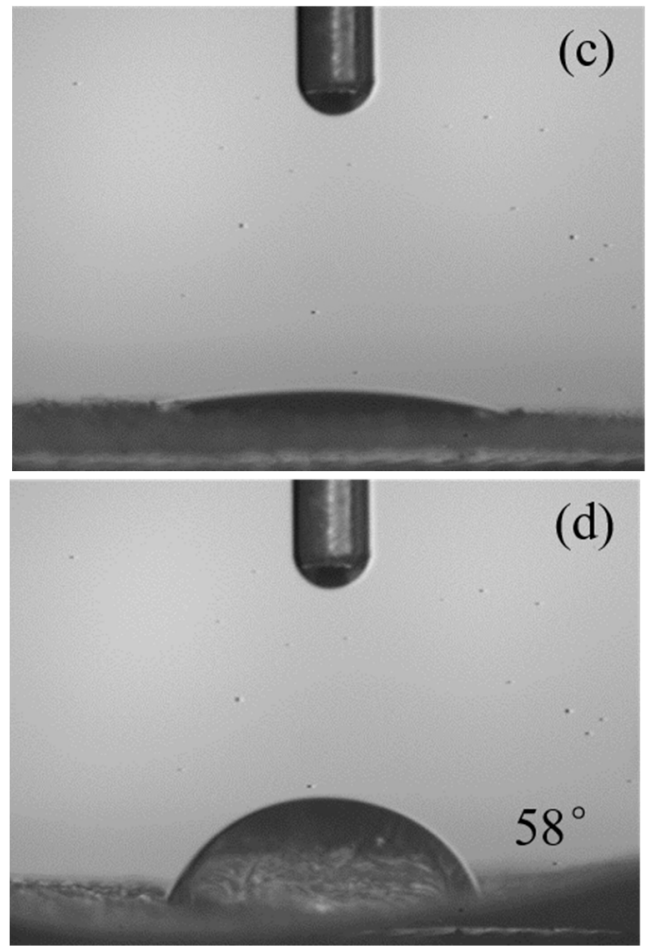

Figure 2. Pore size distribution of (a) PI and (b) PI/cellulose separator and water contact angle micrograph of (c) PI and (d) $\mathrm{PI} /$ cellulose separator.

\subsection{Thermal stability}

The PI battery separator paper was cut into a circular shape with a diameter of $25 \mathrm{~mm}$. After heat treatment at $100^{\circ} \mathrm{C}$, $150{ }^{\circ} \mathrm{C}$, and $200{ }^{\circ} \mathrm{C}$, the diameter of the separator papers are measured to characterize the thermal stability of the separators. The images of the separators at different temperatures were shown in Figure 3. 

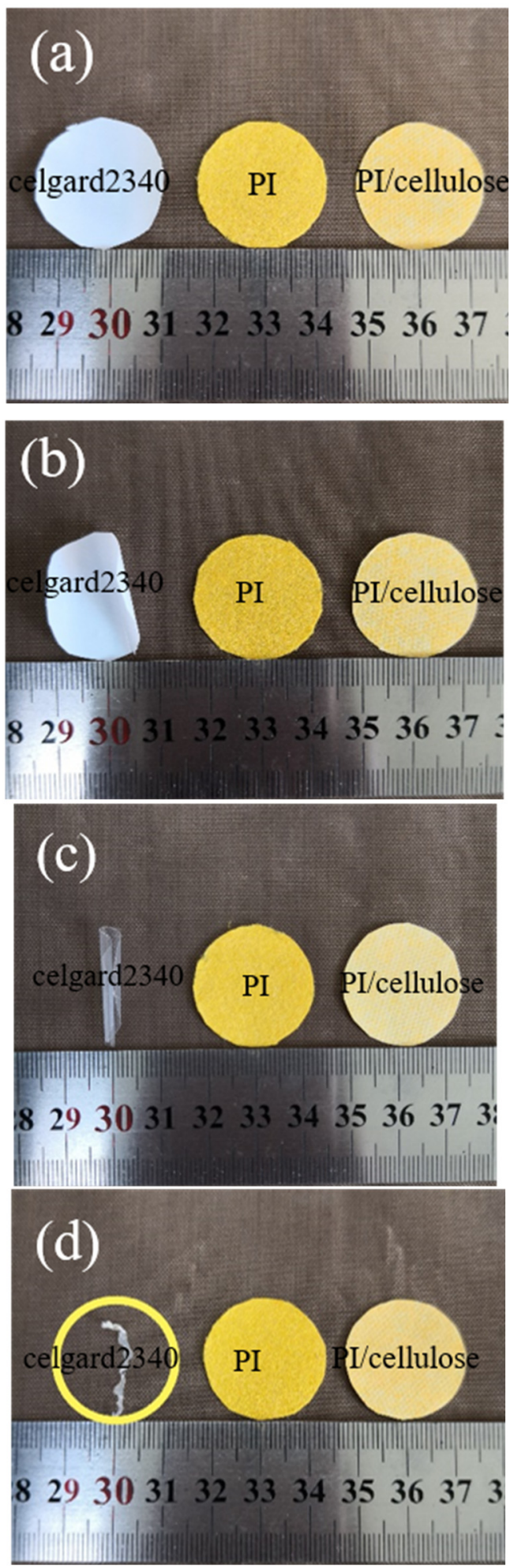

Figure 3. Images of commercial celgard 2340, PI separator, and PI/cellulose separator, at (a) room temperature, (b) $100^{\circ} \mathrm{C}$ for $30 \mathrm{~min}$, and (c) at $150{ }^{\circ} \mathrm{C}$ for $30 \mathrm{~min}$, (d) at $200^{\circ} \mathrm{C}$ for 30 min.

Figure 3 shows that after heat treatment at $100^{\circ} \mathrm{C}$, both the PI separator and the PI/cellulose separator did not shrink, and the celgard 2340 became distorted, however, there was no significant shrinkage. When the heating temperature rises to $150{ }^{\circ} \mathrm{C}$, the celgard 2340 separator was shrunk by $40 \%$ in the shrinkage direction, and the separator was almost melted at $200{ }^{\circ} \mathrm{C}$. However, the PI separator and the $\mathrm{PI} /$ cellulose separator still have no shrinkage. The PI/cellulose separator exhibits enhanced thermal stability compared to the celgard 2340 separator.

\section{CONCLUSIONS}

Compared to commercial celgard 2340 separator, the electrolyte absorption amount of PI/cellulose composite separator was $108.72 \%$, increasing by $80.5 \%$. PI/cellulose composite separator possesses enhanced electrolyte absorption. The commercial celgard 2340 separator is crimped at $100{ }^{\circ} \mathrm{C}$ and the celgard 2340 separator is shrunk at $150{ }^{\circ} \mathrm{C}$. The PI separator and PI / cellulose separator did not shrink at $200{ }^{\circ} \mathrm{C}$, indicating excellent thermal stability, demonstrating its promising application as lithium battery separator.

\section{REFERENCES}

1. Wang H, Wang T, Yang S, et al. Preparation of thermal stable porous polyimide separators by phase inversion process for lithium-ion battery [J]. Polymer, 54(23):6339-6348(2013).

2. Zhang B, Wang Q, Zhang J, et al. A superior thermostable and nonflammable composite separator towards high power battery separator[J]. Nano Energy, 10(1):277-287(2014).

3. Shayapat J, Chung O H, Park J S. Electrospun polyimide-composite separator for lithium-ion batteries[J]. Electrochimica Acta, 170:110121(2015).

4. Wang Y, Wang S, Fang J, et al. A nano-silica modified polyimide nanofiber separator with enhanced thermal and wetting properties for high safety lithium-ion batteries[J]. Journal of Separator Science, 537(2017).

5. Liang X, Yang Y, Jin X, et al. The high performances of $\mathrm{SiO}_{2} / \mathrm{Al}_{2} \mathrm{O}_{3}$-coated electrospun polyimide fibrous separator for lithium-ion battery[J]. Journal of Separator Science, 493:1-7(2015).

6. Shi C, Zhang P, Huang S, et al. Functional separator consisted of polyimide nonwoven fabrics and polyethylene coating layer for lithium-ion batteries [J]. Journal of Power Sources, 298:158-165(2015). 